Journal of Animal and Veterinary Advances 11 (11): 1873-1875, 2012

ISSN: $1680-5593$

(C) Medwell Journals, 2012

\title{
A Rapid and Cost Effective Method for Genotyping Two Types of GHR Mutations in Sex-Linked Dwarf Chicken
}

\author{
${ }^{1,2}$ Yan Wang, ${ }^{1,2} \mathrm{Hao} \mathrm{Qu},{ }^{1,2}$ Cheng-Long Luo, ${ }^{1,2} \mathrm{Jie}$ Wang and ${ }^{1,2}$ Ding-Ming Shu \\ ${ }^{1}$ Guangdong Academy of Agricultural Sciences, Institute of Animal Science, \\ ${ }^{2}$ State Key Laboratory of Livestock and Poultry Breeding, Dafeng 1st Street One, \\ Wushan, Tianhe District, 510640 Guangzhou, Guangdong, People's Republic of China
}

\begin{abstract}
Large deletion or mutation at the exon 10 and 3' untranslated region of Growth Factor Receptor (GHR) gene results in dwarf chicken and the common method for detecting mutation is direct PCR amplification. But since, the target fragment is large, the amplification is inefficient and unreliable and this process consumes a lot of expensive specific DNA polymerases. Researchers focused on the point mutation C146280A in the deletion fragment and set up a new method to detect large deletion and point mutation in this region. Comparing with direct PCR this new method which can detect both large deletion and point mutation with regular low-cost DNA polymerases is more stable, reliably and cheapness.
\end{abstract}

Key words: GHR, sex-linked dwarf chicken, genotyping, mutation, stable

\section{INTRODUCTION}

Sex-linked dwarf chicken has a smaller body size and its weight is only $60-70 \%$ of normal chicken, saving $20 \%$ feed compared with that for normal chicken. Thus, sex-linked dwarf chicken is best for hybrid production because of lower cost and higher economic benefit. It has been reported that the mutation in Growth Factor Receptor $(G H R)$ gene located in the short arm of $\mathrm{Z}$ chromosome results in dwarf chicken phenotype which is sex-linked recessive inheritance (Burnside et al., 1991; Agarwal et al., 1994; Tanaka et al., 1996).

Mutations in chicken GHR gene affect the phenotypes (Tahara et al., 2009). It has been reported that large deletion of exon 10 and 3' UTR (Untranslated Region), G679T and T335C mutation in coding region, two $\mathrm{T}$ to $\mathrm{C}$ mutations with $352 \mathrm{bp}$ from coding region $(352+2)$ result in the dwarf phenotype (Agarwal et al., 1994; Huang et al., 1993; Duriez et al., 1993; Hull et al., 1999).

The large deletion of exon 10 and $3^{\prime}$ UTR leads to a loss of 27 highly conserved amino acids in GHR extracellular domain, resulting in dwarf chicken phenotype (Agarwal et al., 1994). RFLP analysis revealed that $6.0 \mathrm{~kb}$ band from exon 10 and 3' UTR region of wild-type chicken was replaced by $4.3 \mathrm{~kb}$ band in dwarf chicken (Agarwal et al., 1994) and the common method for detecting mutation is direct PCR amplification
(Halabian et al., 2008; Qiong et al., 2011). But since, the target fragment is large, the amplification is inefficient and unreliable and this process consumes a lot of expensive specific DNA polymerases.

Researchers studied the large deletion of exon 10 and 3' UTR (Type I mutant) in GHR gene to look for an easy, low-cost and efficient molecular detecting method. A pair of primers that are relatively easy to amplify were designed within Type I mutation and in the meanwhile they also worked for detection of $\mathrm{Cl} 46280 \mathrm{~A}$ point mutation in $3^{\prime}$ UTR (Type $\Pi$ mutant) (Ouyang et al., 2008). Type I mutant of GHR gene is the deletion of coding region from 1744-3526 bp with a total loss of $1781 \mathrm{bp}$ which is located in exon 10 and 3 ' UTR. Type II mutant is at $1131 \mathrm{bp}$ of type I deletion fragment with $\mathrm{C}$ to $\mathrm{A}$ mutation which results in Hae III-RELP polymorphism.

\section{MATERIALS AND METHODS}

DNA preparation: In this study, all chickens came from the Guangdong Academy of Agricultural Sciences. Genomic DNA was isolated from blood samples of all chickens using a standard phenol:phenol/chloroform purification-based protocol.

Primers: The primers were designed using two end sequences of the deletion fragment in GHR gene Type I mutant as templates P1-F/R (P1-F:5'-GGCAGAAA-

Corresponding Author: Ding-Ming Shu, Animal Breeding and Genetics Group, Guangdong Academy of Agricultural Sciences, Institute of Animal Science, Dafeng 1st Street One, Wushan, Tianhe District, 510640 Guangzhou, Guangdong, China 


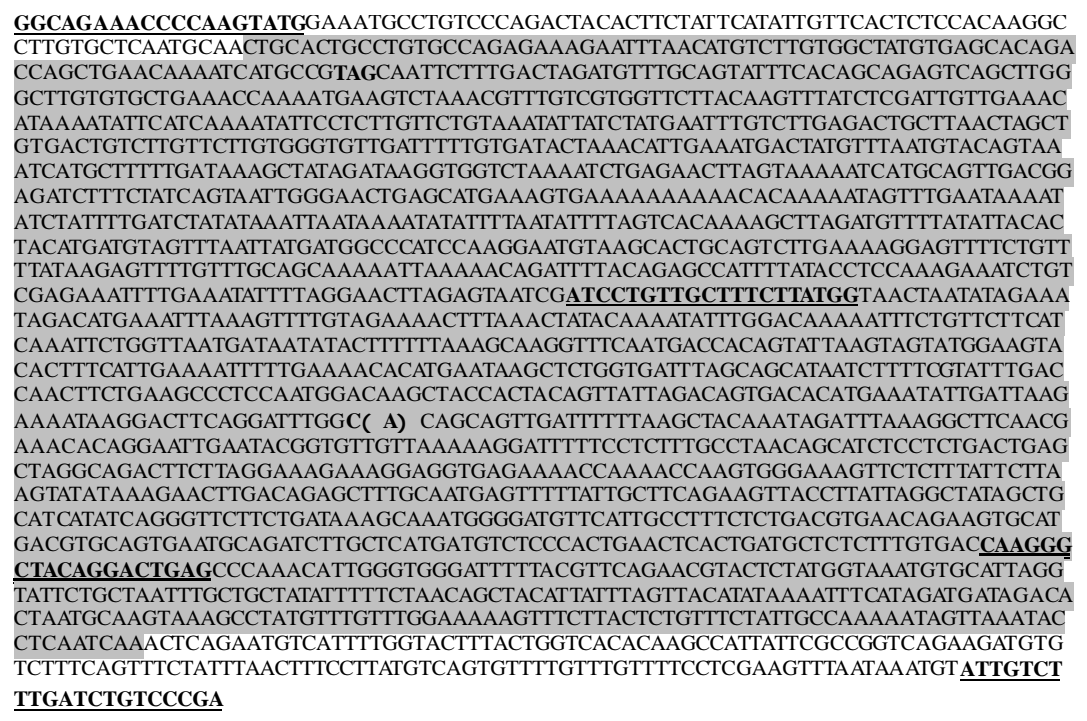

Fig. 1: The expected sequence of amplification product of $\mathrm{P} 1-\mathrm{F} / \mathrm{R}$ and $\mathrm{P} 2-\mathrm{F} / \mathrm{R}$. The framed sequence is the stop code of chicken GHR gene. The sequences underlined are P1-F/R primers. The sequences double-underlined are P2-F/R primers. The shadowed sequence is the sequence lost in Type I mutant. The nucleotide with brackets is point mutation in Type II mutant (C146280A)

CCCCAAGTAT G-3', P1-R: 5'-TCGGGACAGAT-CAAA GACAAT-3'). The two end sequences of Type II mutant (reference to AC187590 of GeneBank) were used as templates for design of primers P2-F/R (P2-F: 5'-ATCCTGT TGCTTTCTTATGG-3',P2-R: 5'-CTCAGTCCTGTAGCCC TTG-3'). The sequence of expected amplification product was shown in supplemental Fig. 1.

The forward primers of P1-F/R and P2-F/R (P1-F and $\mathrm{P} 2-\mathrm{F}$, respectively) were diluted to $10 \mu \mathrm{M}$ and mixed with equal volumes of upstream primers P3-F. P1-R and P2-R were diluted to $10 \mu \mathrm{M}$ and mixed with equal volumes of upstream primers $\mathrm{P} 3-\mathrm{R}$. The $\mathrm{P} 3-\mathrm{F} / \mathrm{R}$ primers were used as a new pair of primers for later experiment.

PCR amplification: The total volume of PCR reaction was $25 \mu \mathrm{L}$ with DNA template $1 \mu \mathrm{L}, 2 \times$ PCR Reaction Mix $12.5 \mu \mathrm{L}$, each of $10 \mu \mathrm{MP} 3-\mathrm{F} / \mathrm{R}$ forward and reverse primers $1 \mu \mathrm{L}$, Taq DNA polymerases (Dongsheng, Guangzhou, China) $0.625 \mathrm{U}, \mathrm{ddH}_{2} \mathrm{O} 8.25 \mu \mathrm{L}$. The PCR conditions were preheating at $94^{\circ} \mathrm{C}$ for $3 \mathrm{~min}$ followed by 33 cycles of $94^{\circ} \mathrm{C}$ for $30 \mathrm{sec}, 56^{\circ} \mathrm{C}$ for $30 \mathrm{sec}$ and $72^{\circ} \mathrm{C}$ elongation for $45 \mathrm{sec}$ with additional elongation at $72^{\circ} \mathrm{C}$ for $10 \mathrm{~min}$. The products were kept at $4^{\circ} \mathrm{C}$. The PCR were subjected to $1.5 \%$ agarose gel electrophoresis.

\section{RESULTS AND DISCUSSION}

The results of PCR amplification using the $\mathrm{P} 3$ primers are shown in Fig. 2. Three genotypes of Type I mutant

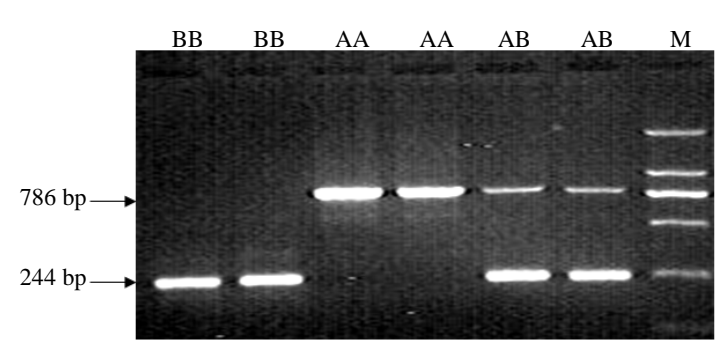

Fig. 2: The PCR amplification product with $\mathrm{P} 3$ primers by electrophoresis (1.5\% agarose gel). $\mathrm{AA}, \mathrm{AB}$ and $\mathrm{BB}$ represent the three genotypes of Type $\mathrm{I}$ mutant. $\mathrm{M}$ is the DNA marker (DL 2000)

were AA (a band of $786 \mathrm{bp}$ ) stands for homozygous wildtype alleles, BB (a band of $244 \mathrm{bp}$ ) represents for homozygous dwarf alleles and $\mathrm{AB}$ (two bands of 786 and $244 \mathrm{bp}$ ) stands for the heterozygous wild-type and dwarf alleles.

Currently, the detection of the mutation in GHR gene was achieved by direct PCR Method. Since, the target band is $>2 \mathrm{~kb}$ (Burnside et al., 1991; Tahara et al., 2009), the amplification efficiency is low and unstable in addition to consumption of expensive specific DNA polymerases. So, the test cost is high while the efficiency is low. The results of PCR amplification using the P3 primers showed that the band of $786 \mathrm{bp}$ can reply the large deletion band (1781 bp) because the $786 \mathrm{bp}$ band located in the deletion band. So, researchers using the P3 


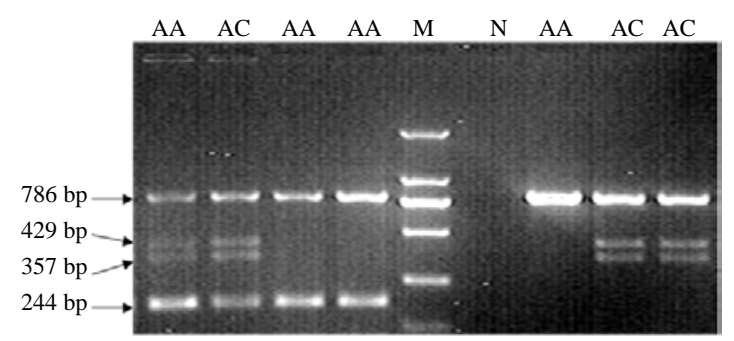

Fig. 3: The endonuclease Hae III digestion of PCR products from amplification with $\mathrm{P} 3$ primers $(1.5 \%$ agarose). AC and AA stand for two genotypes of Type II mutant. M is the DNA marker (DL 2000)

primers to identify the large deletion mutation not only convenient and easier but also does not need expensive LA amplification polymerase.

P3-F/R primers for detecting Type I mutant can be used for detecting Type II mutant too. The single nucleotide mutation C146280A Type II mutant can be recognized by endonuclease Hae III. Further analyses of this mutation were carried out using the Restriction Fragment Length Polymorphism (RFLP) Method. Type II mutant was determined by two alleles. $\mathrm{C}$ is the allele with the endonuclease recognition site and $\mathrm{A}$ is the wild-type allele the band of $244 \mathrm{bp}$ is the product of Type I mutant (Fig. 3).

Comparing with direct PCR this new method which can detect both large deletion and point mutation with regular low-cost DNA polymerases is more stable, reliably and cheapness.

\section{CONCLUSION}

In this study, the method researchers presented can detect the large deletion and C146280A point mutation in GHR gene. It is more convenient, easier, more stable and doesn't need expensive LA amplification polymerase which in turn lowers the test cost and improves the efficiency.

\section{ACKNOWLEDGEMENTS}

This study is supported by two grants. One is from the National Natural Science Foundation of China (Grant No. U0831003). The other is from the National Basic Research Program of China (Grant No.: 2010CB735701).

\section{REFERENCES}

Agarwal, S.K., L.A. Cogburn and J. Burnside, 1994. Dysfunctional growth hormone receptor in a strain of sex-linked dwarf chicken: Evidence for a mutation in the intracellular domain. J. Endocrinol., 142: 427-434.

Burnside, J., S.S. Liou and L.A. Cogburn, 1991. Molecular cloning of the chicken growth hormone receptor complementary deoxyribonucleic acid: Mutation of the gene in sex-linked dwarf chickens. Endocrinology, 128: 3183-3192.

Duriez, B., M.L. Sobrier, P. Duquesnoy, M. TixierBoichard and E. Decuypere et al., 1993. A naturally occurring growth hormone receptor mutation: In vivo and in vitro evidence for the functional importance of the WS motif common to all members of the cytokine receptor superfamily. Mol. Endocrinol., 7: 806-814.

Halabian, R., N. Morad Pasha Eskandari, N. Mohammad Reza, M. Ali Reza Heravi, H. Seyed Abolfazl and Q. Saber, 2008. Characterization of SNPs of bovine prolcatin gene of Holstein cattle. Biotechnology, 7: 118-123.

Huang, N., L.A. Cogburn, S.K. Agarwal, H.L. Marks and J. Burnside, 1993. Overexpression of a truncated growth hormone receptor in the sex-linked dwarf chicken: Evidence for a splice mutation. Mol. Endocrinol., 7: 1391-1398.

Hull, K.L., J.A. Marsh and S. Harvey, 1999. A missense mutation in the GHR gene of Cornell sex-linked dwarf chickens does not abolish serum GH binding. J. Endocrinol., 161: 495-501.

Ouyang, J.H., L. Xie, Q. Nie, C. Luo, Y. Liang, H. Zeng and X. Zhang, 2008. Single Nucleotide Polymorphism (SNP) at the GHR gene and its associations with chicken growth and fat deposition traits. Br. Poult. Sci., 49: 87-95.

Qiong, W., F. Chao, L. Wu-Jun, F. Yi and Y. Shi-Gang, 2011. A novel mutation at exon 4 of IGF-1 gene in three indigenous goat breeds in china. Asian J. Anim. Vet. Adv., 6: 627-635.

Tahara, K., A. Tsukada, T. Hanai, K. Okumura and $\mathrm{K}$. Yamada et al., 2009. Identification of two types of growth hormone receptor mutations in two stains of sex-linked dwarf chickens. J. Poult. Sci., 46: 249-256.

Tanaka, M., Y. Hayashida, K. Sakaguchi, T. Ohkubo, M. Wakita, S. Hoshino and K. Nakashima, 1996. Growth hormone-independent expression of insulin-like growth factor I messenger ribonucleic acid in extrahepatic tissues of the chicken. Endocrinology, 137: 30-34. 